\title{
DISTRIBUIÇÃO DE RENDA E CRESCIMENTO ECONÔMICO NO BRASIL: UMA ANÁLISE ECONOMÉTRICA DO PERÍODO 1976-2010
}

\author{
Moisés Pais dos Santos* \\ Marina Silva da Cunha \\ Sérgio Ricardo de Brito Gadelha
}

\begin{abstract}
Resumo
O modelo brasileiro de crescimento econômico predominante se caracteriza por baixas taxas de crescimento, grau reduzido de dinamismo e intensa desigualdade social. Esta pesquisa se norteia pela seguinte questão: de que forma e em que magnitude a distribuição de renda e o crescimento econômico brasileiro tem se inter-relacionado ao longo do período 1976-2012? O objetivo deste trabalho é estudar as relações de longo prazo entre a distribuição de renda e crescimento econômico no Brasil. Tanto a modelagem VEC quanto os modelos autorregressivos de defasagens distribuídas (ADLs) sugeriram a existência, no sentido de Granger, de bicausalidade da desigualdade para o crescimento e do crescimento para a desigualdade.
\end{abstract}

Palavras-chave: Crescimento econômico, desigualdade, séries temporais.

\section{Abstract}

The Brazilian model of economic growth is characterized by low growth rates, low level of dynamism and intense social inequality. This research is guided by the following question: in what way and to what extent the distribution and Brazilian economic growth has interrelated over the period 1976-2012? The objective of this work is to study the long-term relationships between income distribution and economic growth in Brazil. Both VEC modeling as the autoregressive models of distributed lags (ADLs) suggested the existence, in the sense of Granger, causality of inequality for growth and growth for inequality. In Brazil, income inequality hampers economic grouth because of the inertia of inequality and also economic growth is revealed as an important tool for reducing income inequality.

Keywords: economic growth, inequality, times series

JEL: O1

\section{Introdução}

A forma de condução da política econômica brasileira tem despertado a atenção de pesquisadores para a realização de diversos estudos, principalmente pelo fato de que o modelo de crescimento econômico predominante se caracteriza por taxas baixas de crescimento e grau reduzido de dinamismo em um cenário econômico permeado pela forte concentração de renda, apesar da sua tendência

\footnotetext{
* Universidade Estadual de Maringá (PCE/ UEM)
} 
de amenização. No século XX, destacou-se a contribuição de Kuznets (1955) que analisou a relação entre desigualdade de renda e o desenvolvimento econômico mediante um modelo dual cuja hipótese básica era que a desigualdade de renda se elevaria no curto prazo e o desenvolvimento econômico se encarregaria de reduzi-la, configurando-se assim, uma relação com o formato de U-invertido entre essas variáveis.

Estudos mais recentes sobre o tema procuram também abordar a capacidade do capital humano e da política econômica afetar a concentração de renda. Piketty (2014), por exemplo, defende que a dinâmica da distribuição de riqueza ora tende para a convergência e ora tende para a divergência, inexistindo qualquer processo natural capaz de impedir a prevalência das forças desestabilizadoras e promotoras da desigualdade. No Brasil, a divulgação dos dados do Censo de 1970 despertou um longo debate sobre as causas da concentração de renda da década de 1960. Diversos estudos, tais como os de Hoffmann e Duarte (1972), Singer (1975), Fishlow (1975), Furtado (1982), Tavares e Assis (1985) têm atribuído esse feito à política econômica adotada pelos governos da época, principalmente, os primeiros a governar o país depois de instaurado o golpe militar de 1964.

A discussão sobre os anos de 1960 tem como uma das características, a divisão em duas correntes distintas e bem definidas. De um lado, autores como Langoni (1973) e Senna (1976) se basearam no modelo de capital humano e relacionaram a deterioração na distribuição de renda ao ritmo intenso de crescimento econômico no final da década de 1960. De outro, pesquisadores como Hoffmann e Duarte (1972), Fishlow (1975) rejeitam o modelo de capital humano e estabelecem relações entre a política econômica e a concentração de renda.

As produções científicas mais recentes sobre a relação entre distribuição de renda e crescimento econômico têm se baseado na teoria do crescimento endógeno ${ }^{1}$. As pesquisas que consideram os efeitos da desigualdade sobre o crescimento (positivos e negativos) em contrapartida àquelas que estudam os efeitos do crescimento sobre a desigualdade inspirados em Kuznets (1955) têm sido mais comum. Enquanto Galor e Zeira (1993), Alesina e Rodrik (1991), Barro (2000), Shin (2012), Iradian (2005) desenvolveram modelos empíricos para explicar os efeitos da desigualdade de renda no crescimento, Zhou e Li (2011), Risso, Punzo e Carrera (2013) estudaram os efeitos do crescimento sobre a desigualdade. Diferentemente desses dois grupos distintos de investigação científica, Lin Huang e Yeh (2009) inovam ao estudar simultaneamente os dois

\footnotetext{
${ }^{1}$ Conforme Jones (1995), essa teoria sugere que mudanças permanentes em determinadas variáveis políticas têm efeitos permanentes sobre a taxa de crescimento econômico. A teoria resulta de dois modelos que contrastam com o modelo proposto por Solow (1956) em que a presença do crescimento de longo prazo depende fundamentalmente do progresso tecnológico exógeno. Inicialmente foi desenvolvido o modelo do tipo "AK" por Romer (1986, 1987) e Lucas (1988). Devido à insatisfação com a performance empírica desses tipos de modelos, a literatura do crescimento endógeno passou a adotar modelos que explicassem o crescimento de longo prazo focando no progresso tecnológico e na Pesquisa e Desenvolvimento, como os de Romer (1990), Grossman e Helpman (1991) e Aghion e Howitt (1992). Nesses, progresso tecnológico é resultado da busca por inovações na maximização do lucro individual.
} 
efeitos, variando a causalidade, partindo tanto da desigualdade para o crescimento quanto do crescimento para a desigualdade.

Em termos de política econômica, o crescimento econômico e a distribuição de renda são objetivos importantes nas economias. Na época do "milagre" econômico se verificou um conflito de objetivos numa combinação de intenso crescimento e aumento da concentração de renda. Apesar da implantação de programas de estabilização de preços e de transferência de renda no Brasil, os indicadores econômicos e sociais sugerem elevada concentração de renda e baixas taxas de crescimento da economia brasileira. Diante disso, esta pesquisa se norteia pela seguinte questão: de que forma e em que magnitude a distribuição e o crescimento econômico brasileiro têm se inter-relacionado ao longo do período 1976-2012? A hipótese do trabalho é que existe uma relação de longo prazo entre distribuição e crescimento e que enquanto o crescimento econômico afeta positivamente a distribuição, essa, por sua vez, afeta negativamente o crescimento. Portanto, a relação de longo prazo entre crescimento e desigualdade é bicausal.

Em vista do questionamento levantado, o objetivo geral do presente trabalho é estudar as relações de longo prazo entre a distribuição de renda e crescimento econômico no Brasil, durante o período 1976-2012, mediante a abordagem de séries temporais. A justificativa para se realizar esse estudo se baseia no fato de ainda haver ausência de pesquisas que tratem especificamente da relação entre desigualdade e crescimento bem como dos mecanismos de transmissão dos efeitos da desigualdade sobre a economia. Além de preencher essa lacuna na literatura sobre o tema, a pesquisa também se justifica pelo fato de que, ao longo das últimas quatro décadas, a economia brasileira se caracteriza pela predominância de baixas taxas de crescimento econômico e elevado patamar de desigualdade de renda. Ambas as características denunciam um viés em relação aos principais objetivos de quaisquer economias: crescimento econômico e distribuição de renda.

O presente estudo contribui à literatura sobre o tema por encontrar os seguintes resultados para o caso brasileiro: i) existência de relação de longo prazo entre desigualdade de renda e crescimento, mediante a modelagem VEC e modelos autorregressivos com defasagens distribuídas; ii) a desigualdade de renda prejudica o crescimento por causa da inércia da desigualdade; iii) da mesma forma que a desigualdade precede temporalmente o crescimento, o desempenho econômico também precede a desigualdade de renda; iv) alguns dos canais de transmissão dos efeitos da desigualdade sobre o crescimento como por exemplo, redistribuição e diferencial de fertilidade, não podem ser considerados importantes para explicar o efeito negativo da desigualdade sobre o crescimento no Brasil; v) o crescimento econômico consiste num instrumento importante para a redução da desigualdade de renda.

Além dessa introdução, o trabalho é composto por outras quatro seções. A segunda parte deste trabalho faz uma breve revisão de literatura sobre desigualdade e crescimento, destacando os objetivos, métodos e principais resultados. A terceira parte descreve a metodologia econométrica de séries temporais além da fonte dos dados e variáveis utilizadas. Na quarta seção são apresentados os principais resultados obtidos mediante os modelos estimados e 
testes realizados. A quinta e última seção ficou reservada para as considerações finais.

\section{A literatura}

Esta breve revisão de literatura tem o objetivo de fornecer uma síntese das principais contribuições teóricas e empíricas sobre a relação entre desigualdade e crescimento. As contribuições mais tradicionais sobre o tema polarizavam na discussão de uma relação linear positiva ou negativa enquanto os trabalhos mais recentes privilegiam a relação não linear dos efeitos da desigualdade sobre o crescimento econômico. Dessa forma, têm-se os efeitos da desigualdade de renda sobre o crescimento econômico que podem ser positivos, negativos, tanto nas relações lineares quanto nas relações não lineares. Por outro lado, estudos inspirados em Kuznets (1955) têm tratado a medida da concentração de renda como sendo a variável dependente e o crescimento econômico como a variável explicativa.

Assim, inicialmente, há trabalhos que estudam os efeitos positivos da desigualdade sobre o crescimento. Nesses os pesquisadores têm buscado suporte teórico nos modelos clássicos, segundo os quais o crescimento econômico depende principalmente da taxa pela qual as nações acumulam recursos produtivos, portanto, da taxa de poupança agregada. A abordagem clássica originou-se de Smith (1776) e foi interpretada e desenvolvida por Keynes (1920), Kaldor (1957) e Bourguignon (1981). Conforme essa abordagem, a taxa de poupança da economia é uma função crescente da riqueza e da desigualdade. Considerando uma economia com indivíduos cuja propensão marginal a poupar é mais elevada, acréscimos na taxa de poupança agregada aumentam a acumulação de capital e, consequentemente, estimulam o crescimento econômico (Galor \& Moav, 2004).

Nessa abordagem, a questão distributiva afeta o crescimento somente se a propensão a poupar dos indivíduos variar com a renda e/ ou a riqueza (Risso, Punzo \& Carrera, 2013). Nos países desenvolvidos, onde a taxa de poupança dos indivíduos ricos é maior que a dos pobres, uma política redistributiva de renda de pessoas ricas para as pessoas pobres reduz a taxa de poupança agregada da economia causando a desaceleração do crescimento econômico. Outra causa da redução do crescimento econômico é que a redistribuição de renda pode diminuir o incentivo para que os mais ricos trabalhem mais arduamente. Como resultado da visão clássica, infere-se que enquanto a distribuição igualitária da renda desaceleraria o crescimento econômico, a desigualdade o estimularia (Shin, 2012). Estudos como os de Okun (1975), Bourguignon (1981), Benabou (1996), Li e Zou (1998), Aghion e Howitt (1998) e Forbes (2000) têm abordado o efeito positivo da desigualdade de renda sobre o crescimento econômico, mediante uma relação linear de causalidade.

No que tange aos efeitos negativos da desigualdade sobre o crescimento econômico, modelos teóricos abordam os canais de transmissão dos efeitos indiretos da desigualdade. Os principais canais seriam a redistribuição de renda através da tributação, os mercados imperfeitos de crédito, o conflito social e o diferencial de fecundidade entre ricos e pobres. Os modelos com imperfeições no mercado de crédito foram desenvolvidos por Alesina e Rodrik (1991), Persson e Tabellini (1994). Quanto ao canal da redistribuição de renda foram 
desenvolvidos os modelos de economia política que, por sua vez, estão fundamentados no teorema do eleitor mediano; destacando-se as contribuições de Black (1948), Ehrhart (2009), Alesina e Rodrik (1991), Persson e Tabellini (1994). Os modelos de conflito social são descritos em Alesina e Perotti (1996), Keefer e Knack (2002) enquanto que os modelos que abordam a conexão entre diferencial de fertilidade e desigualdade são abordados em Perotti (1996) e Ehrhart et al. (2009).

\section{Abordagem metodológica}

Para analisar a relação entre distribuição de renda e crescimento econômico este trabalho utilizou alguns dos procedimentos e testes para análise de dados de séries temporais. Inicialmente, foi analisada a estacionariedade das séries com base em testes de raiz unitária. Elliot, Rottemberg e Stock (1996) e NG e Perron (2001) propuseram os testes modificados de Dickey-Fuller (ADFGLS) e de Phillips-Perron $\left(\overline{\left.M Z_{\alpha}^{G L S}\right)}\right.$ cuja finalidade é verificar a estacionariedade das séries. Esses testes se caracterizam por superar os problemas de baixo poder estatístico e distorções de tamanho dos testes tradicionais de Dickey e Fuller (1979), Said e Dickey (1984) e de Phillips e Perron (1988).

As modificações nos testes tradicionais se justificam pelo fato de que a extração de tendência em séries temporais usando Mínimos Quadrados Ordinários (MQO) é ineficiente e também, devido à importância de uma seleção apropriada para a ordem de defasagem do termo aumentado, de modo a obter uma melhor aproximação para o verdadeiro processo gerador de dados.

Diante dessas preocupações, Elliot, Rottemberg e Stock (1996) propõem usar o estimador Mínimos Quadrados Generalizados (MQG) ao invés de MQO para extrair a tendência estocástica da série. Os critérios de informação de Akaike (AIC) e de Schwarz (SIC), segundo NG e Perron (2001), tendem a selecionar baixos valores para a defasagem $k$, quando se tem uma raiz negativa e próxima da unidade no polinômio de médias móveis da série, conduzindo os testes de raízes unitárias a fortes distorções.

Na sequência, foram estimados os modelos autorregressivos vetoriais (VAR) e de defasagens distribuídas (ADL), os quais só podem ser estimados para séries estacionárias, em nível ou nas suas diferenças. Posteriormente, foram realizados os testes de cointegração de Engle-Granger e de J ohansen (1988).

\subsection{Abordagem VAR e ADL}

O modelo VAR é útil para analisar as inter-relações entre múltiplas séries temporais, partindo do pressuposto de que todas as variáveis são determinadas endogenamente num sistema de equações (Enders, 2004). Exemplificando com apenas duas variáveis, esse modelo pode ser expresso mediante o sistema de equações que se segue:

$$
\begin{aligned}
& W_{\mathrm{t}}=\alpha_{1} W_{\mathrm{t}-1}+\alpha_{2} Z_{\mathrm{t}-1}+\mathrm{e}_{\mathrm{t}}^{\mathrm{w}} \\
& \mathrm{Z}_{\mathrm{t}}=\beta_{1} W_{t-1}+\beta_{2} \mathrm{Z}_{\mathrm{t}-1}+e_{\mathrm{t}}^{z}
\end{aligned}
$$


Onde $e^{w_{t}}$ e $e^{z_{t}}$ são processos ruído branco e não correlacionados. Alternativamente, tem-se a seguinte equação reduzida:

$$
\mathrm{y}_{\mathrm{t}}=\psi \mathrm{y}_{\mathrm{t}-1}+e_{\mathrm{t}}
$$

Sendo: $\mathrm{y}_{\mathrm{t}}=$ vetor das variáveis endógenas $\mathrm{w}_{\mathrm{t}} \mathrm{e} \mathrm{zt} ; \Psi=$ matrizes dos coeficientes a serem estimados; $\varepsilon_{\mathrm{t}}=$ vetor de inovações.

A abondagem VAR caracteriza-se pelo fato de ser um modelo ateórico e também, pela geração de coeficientes individuais que não são fáceis de serem interpretados diretamente. Em contrapartida, sua metodologia envolve ferramentas importantes, tais como, a análise de decomposição de variância e as funções de impulso-resposta que podem ser utilizadas após a estimação dos parâmetros do modelo VAR. A abordagem de Granger (1989) sobre a causalidade é no sentido de analisar quanto o presente pode ser explicado por valores passados se a inclusão de valores defasados no modelo pode melhorar o seu poder de predição.

A dependência de uma variável em relação à outra nem sempre é instantânea. No caso do estudo da relação entre distribuição de renda e crescimento econômico, um aumento na concentração de renda no período atual pode afetar negativamente ou positivamente o crescimento econômico em períodos futuros. Da mesma forma, um aumento na taxa de crescimento econômico no período atual pode afetar negativamente ou positivamente a concentração de renda em períodos posteriores. Sendo assim, a reação da variável dependente em relação à variável explanatória demora certo intervalo de tempo conhecido como defasagem.

Diante da importância do impacto da defasagem para análise da reação das variáveis dependentes foram estimados os modelos ADLs (Autorregressivos de defasagens distribuídas) que, em termos gerais, apresentam a seguinte forma:

$$
y_{t}=\alpha+\beta_{0} x_{t}+\beta_{1} x_{t-1}+\beta_{2} x_{t-2}+\ldots+\beta_{k} x_{t-k}+\theta_{1} y_{t-1}+\theta_{2} y_{t-2}+\ldots+\theta_{i} y_{t-i}+\varepsilon_{t}
$$

Esses modelos se caracterizam pelas defasagens distribuídas com uma defasagem finita de $\mathrm{k}$ períodos.

\subsection{Dados da pesquisa}

Diante da necessidade de se estudar a relação de longo prazo entre distribuição de renda e crescimento econômico, optou-se pela utilização das seguintes variáveis: coeficiente de Gini (Gini) e Produto Interno Bruto real per capita (PIBpc) ${ }^{2}$. Os dados utilizados neste trabalho foram coletados nos sítios eletrônicos do Instituto Brasileiro de Geografia e Estatística (IBGE) e do Instituto de Pesquisa Econômica Aplicada (IPEADATA).

Por sua vez, os dados de concentração de renda foram coletados no IPEADATA cuja fonte primária é a Pesquisa Nacional por Amostra de Domicílios

\footnotetext{
2 Alternativamente, como medida da desigualdade de renda, também foram realizadas simulações (não apresentadas neste trabalho) com o Índice de Theil, porém, os resultados foram bastantes similares àqueles quando se utiliza o Coeficiente de Gini, tanto nos testes de raiz unitária, quebra estrutural, cointegração quanto nas estimativas dos modelos VAR e VEC.
} 
(PNAD/IBGE)3. Para os anos 1991, 1994, 2000 e 2010 utilizou-se a média dos quatro anos anteriores. Sendo assim, foi construído um banco de dados contendo duas séries temporais, PIBpc e Gini, para o período 1976 a 2012. A FIGURA 1 ilustra que essas duas séries temporais exibem tendências distintas. Analisando as duas séries temporais de interesse nesse trabalho, para o período 1976-2012, verifica-se que enquanto a tendência da série Gini decresce ao longo do tempo, a série PIBpc possui tendência ascendente ao longo do tempo.

FIGURA 1 - COEFICIENTE DE GINI E PIB PER CAPITA, BRASIL, 1976-2016

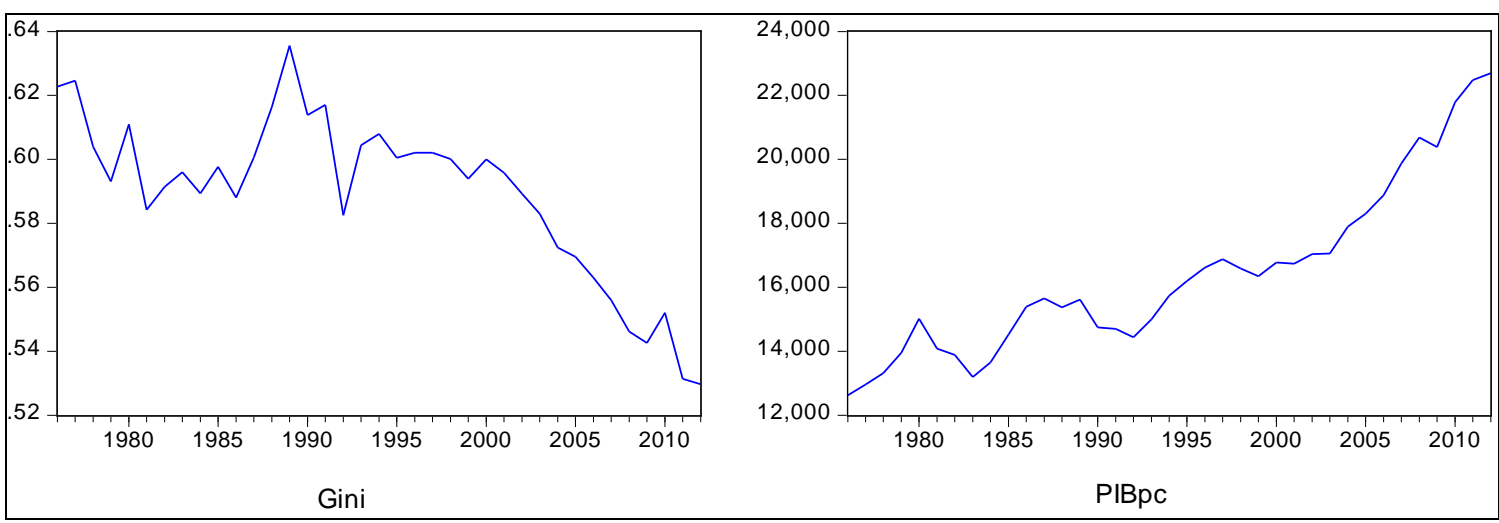

Fonte: IBGE e IPEADATA. Elaboração própria.

A desigualdade de renda no Brasil, apesar de extremamente elevada em relação à maioria dos países do mundo, tem apresentado tendência de redução, em meados da década de 1980, provavelmente em decorrência dos planos de estabilização de preços. Entretanto, o fracasso desses planos resultou em hiperinflação e corroborou para que a concentração revertesse sua tendência atingindo patamares mais elevados no final da década de 1980 que no seu início.

\section{Resultados e discussões}

Inicialmente, foi analisada a estacionariedade das séries que representam a distribuição de renda e o crescimento econômico e, em geral, os quatro testes de raiz unitária com e sem quebra estrutural sugerem a decisão pela ordem de integração um, I(1), tanto para a variável PIBpc quanto para Gini. Esses testes sugeriram a presença de quebra estrutural para os seguintes anos: 1984, 1990 e $2000^{4}$. Os resultados dos testes modificados de raiz unitária Dickey-Fuller ${ }^{5}$ $\left(A D F^{G L S}\right)$ e de Phillips-Perron $\left(\overline{M Z_{\alpha}^{G L S}}\right)$ propostos por Elliot, Rottemberg e Stock

${ }^{3}$ O coeficiente de Gini foi calculado pelo IPEA com base na distribuição da renda domiciliar per capita entre os indivíduos, a partir das respostas à Pesquisa Nacional por Amostra de Domicílios (Pnad/ IBGE).

4 Para cada um desses anos foi criada uma variável binária (d1984, d1990 e d2000), em seguida, estimou-se um modelo VAR considerando essas binárias como sendo variáveis exógenas. Somente a dummy d1984 foi considerada estatisticamente significativa.

5 Valores críticos do teste ADFGLS são (Elliot, Rothenberg \& Stock, 1996); Especificação C, T: $3,77(1 \%) ;-3,19(5 \%)$ e-2,89 (10\%). 
(1996) e NG e Perron (2001) ${ }^{6}$, sem quebra estrutural, indicaram que a variável PIBpc, tanto com constante, como com constante e tendência, não é estacionária, quer seja em nível ou em primeira diferença. Esses mesmos testes sugeriram que a variável Gini, com constante e tendência, seja estacionária em primeira diferença.

Quanto aos testes de raiz unitária com quebra estrutural propostos por Perron (1989) ${ }^{7}$, os resultados indicaram que, para a variável PIBpc, não foi possível rejeitar a hipótese nula de presença de raiz unitária mesmo para os três diferentes tipos de quebra estrutural: constante; tendência; constante e tendência. Já para a variável Gini, foi possível rejeitar a hipótese nula de presença de raiz unitária ao nível de significância de $10 \%$ num modelo de quebra estrutural no ano 2000 e com tendência. De acordo com esse teste, a variável PIBpc foi considerada não estacionária enquanto a variável Gini foi considerada estacionária.

Ademais, conforme os testes de raiz unitária com quebra estrutural endógena propostos por Saikkonen e Lütkepohl (2002) e Lanne, Lütkepohl e Saikkonen $(2002)^{8}$ foi possível rejeitar, ao nível de $1 \%$, a presença de raiz unitária para a variável PIBpc em nível mediante o modelo de quebra estrutural rational shift com constante e tendência, mas não quando o ano de 1981 foi apontado como sendo a data da quebra estrutural endógena. Mediante esse mesmo teste, a variável Gini em nível foi considerada não estacionária quer seja por qualquer um dos três tipos de modelos testados: rational shift, exponential shift e shift dummy. Porém, a variável Gini em primeira diferença foi considerada estacionária mediante o modelo de quebra estrutural rational shift com constante. O ano de 1990 foi apontado como sendo a data da quebra estrutural endógena. Portanto, foi possível concluir que as variáveis são I(1) e, uma vez que as variáveis analisadas não são estacionárias em nível, o modelo apropriado para investigar a relação de causalidade entre distribuição de renda e crescimento econômico deveria ser estimado em primeiras diferenças. No entanto, diante da perda de informação de qualquer relação de longo prazo entre as variáveis em primeira diferença, utiliza-se a análise de cointegração. Se a cointegração para uma formulação particular for estabelecida, recupera-se o mecanismo de correção de erro (MCE) e se estima o modelo VEC. Tal procedimento permite obter, simultaneamente, o comportamento dinâmico de curto prazo das duas variáveis de interesse (causalidade de curto prazo), bem como testar a direção de causalidade entre as duas variáveis no longo prazo.

Tanto o teste de cointegração de Engle-Granger quanto o J ohansen (2000) sugerem que há pelo menos um vetor de cointegração entre Gini e PIBpc. Tomando-se Gini como a variável dependente, não foi possível rejeitar a hipótese nula de que essa variável esteja cointegrada com a variável PIBpc ao nível de significância de 10\%. Entretanto, tomando-se PIBpc como a variável

6 Valores críticos do teste $\overline{M Z} \underset{\propto}{G L S}$ são (Ng \& Perron, 2001); Especificação C, T: -3,42 (1\%); -2,91 (5\%) e-2,62 (10\%).

7 Valores críticos do teste de Perron (1989, 1998); (i) Especificação T: -5,45 (1\%); -4,83 (5\%) e 4,48 (10\%); (ii) Especificação C, T: -6,32 (1\%); -5,59 (5\%) e-5,29 (10\%).

8 Valores críticos do teste de Saikkonen-Lutkepohl (Lanne et al. 2002); Especificação C: -3,48

(1\%); -2,88 (5\%) e-2,58 (10\%); (ii) Especificação C, T: -3,55 (1\%); -3,03 (5\%) e-2,76 (10\%). 
dependente, foi possível rejeitar a hipótese nula, ao nível de significância de $10 \%$, de que essa variável esteja cointegrada com a variável Gini. Além da abordagem VEC, a análise da relação entre desigualdade e crescimento econômico também foi realizada mediante os modelos ADL os quais possibilitam maior flexibilidade no uso das defasagens. Segundo Gadelha (2011), enquanto a modelagem VEC impõe a restrição de que cada variável tenha o mesmo número de defasagens em todas as equações do modelo, os modelos ADL são abrangentes e permitem estudar a dinâmica dos sistemas sem incorrer no viés de omissão de defasagens relevantes.

Assim, a TABELA 1 apresenta os resultados estimados para os modelos VEC e ADL mediante duas especificações diferentes: uma que considera a variável Gini como sendo a variável dependente e outra que toma como variável dependente, o PIB per capita. Uma vez que as séries são integradas de ordem um, os procedimentos foram realizados para as séries nas primeiras diferenças. Para cada uma dessas especificações foram estimados dois modelos, um VAR e outro VEC que inclui o mecanismo de correção de erro (MCE). Também, para cada uma dessas duas especificações diferentes, foram estimados dois modelos ADL: $\operatorname{ADL}(6,3)$ e ADL $(5,8)$.

Uma vez que existe cointegração entre as variáveis Gini e PIBpc, pode-se afirmar que existe uma relação de equilíbrio de longo prazo entre essas variáveis e essa relação também precisa ser inserida no modelo ADL. Estimou-se um modelo ADL $(6,3)^{9}$ com a variável Gini em primeira diferença como a variável dependente e as variáveis explanatórias são a primeira diferença da variável Gini em seis defasagens, o PIB contemporâneo e a sua primeira diferença em três defasagens além do mecanismo de correção de erro (MCE) obtido a partir da regressão de Mínimos Quadrados Ordinários (MQO) das variáveis contemporâneas. O coeficiente de determinação ajustado $\left(\bar{R}^{2}\right)$ sugere que $18 \%$ das variações da variável dependente são explicadas pelas variáveis explanatórias inseridas no modelo.

Constatou-se que as variações da desigualdade em cinco defasagens e variações no PIB per capita em três defasagens foram consideradas estatisticamente significativas para explicar as variações contemporâneas da desigualdade. O sinal do mecanismo de correção de erro (MCE) está de acordo com o esperado e, também, é estatisticamente significativo ao nível de significância de $10 \%$. Seu valor, equivalente a $-0,5473$ sugere que pelo menos $54 \%$ dos erros do modelo são corrigidos no primeiro período.

Seguindo essa mesma abordagem econométrica, também foi estimado um modelo ADL $(5,8)^{10}$ com o PIB em primeira diferença com a variável

\footnotetext{
9 Inicialmente foi estimado um modelo ADL $(6,7)$ tendo Gini como variável dependente e PIB como variável independente, entretanto, ao estimar esse modelo, apesar de um $\mathrm{R}^{2}$ ajustado de 0,2467 e dos sinais da maioria dos parâmetros estarem conforme o esperado, estes não são estatisticamente significativos. Partiu-se então para a segunda melhor opção, ou seja, um modelo ADL $(6,3)$.

10 Para a seleção do número ótimo de defasagens do modelo ADL, utilizou-se o comando ARIMA selection do pacote econométrico E-Views 7.0, iniciando-se com um número de oito (8) defasagens para selecionar o modelo que apresentasse o menor critério de informação de
} 
dependente ${ }^{11}$ e as variáveis explanatórias são a primeira diferença da variável PIBpc de uma até cinco defasagens e a primeira diferença da variável Gini em oito defasagens, além do mecanismo de correção de erro (MCE) obtido a partir da regressão MQO das variáveis contemporâneas. O coeficiente de determinação ajustado $\left(\bar{R}^{2}\right)$ sugere que mais de $61 \%$ das variações da variável dependente são explicadas pelo modelo. Mediante a estatística F, foi possível rejeitar a hipótese nula de que os coeficientes dos parâmetros, conjuntamente, são iguais a zero.

TABELA 1 - RESULTADOS DAS REGRESSÕES PARA OS MODELOS VEC E ADL, BRASIL, 1976-2012

\begin{tabular}{|c|c|c|c|c|c|c|c|c|}
\hline \multicolumn{5}{|c|}{ Variável dependente: $\Delta$ LnGini } & \multicolumn{4}{|c|}{ Variável dependente: $\Delta \mathrm{LnPIBpc}$} \\
\hline Variáveis & VEC & $\begin{array}{c}\text { Teste } \\
\mathrm{t}\end{array}$ & $\begin{array}{l}\mathrm{ADL} \\
(6,3)\end{array}$ & $\begin{array}{c}\text { Teste } \\
\mathrm{t}\end{array}$ & VEC & $\begin{array}{c}\text { Teste } \\
\mathrm{t}\end{array}$ & $\begin{array}{l}\mathrm{ADL} \\
(5,8)\end{array}$ & $\begin{array}{c}\text { Teste } \\
\mathrm{t}\end{array}$ \\
\hline Constante & $-0,0169 *$ & $-1,8$ & $-0,0007$ & $-0,1$ & $-0,0254^{*}$ & $-1,9$ & $0,0327 * * *$ & $-4,95$ \\
\hline$\Delta L n P I B p c_{\mathrm{t}-1}$ & $-0,1034$ & $-1,00$ & $-0,1758$ & $-1,3$ & $0,3203^{* *}$ & - & $-0,4711^{* *}$ & $-2,13$ \\
\hline$\Delta L n P I B p c_{\mathrm{t}-2}$ & - & - & 0,2023 & $-1,6$ & - & - & $-0,2686$ & $-1,39$ \\
\hline$\Delta L n P I B p c_{\mathrm{t}-3}$ & - & - & 0,2689* & -2 & - & - & $-0,1256$ & $-0,69$ \\
\hline$\Delta L n P I B p c_{\mathrm{t}-4}$ & - & - & - & - & - & - & $-0,0072$ & $-0,04$ \\
\hline$\Delta L n P I B p c_{\mathrm{t}-5}$ & - & - & - & - & - & - & $-0,2021$ & $-1,13$ \\
\hline$\Delta$ LnGini & - & - & - & - & - & - & $-0,0127$ & $-0,04$ \\
\hline$\Delta$ LnGinit-1 $_{\mathrm{t}}$ & $-0,1176$ & $-0,7$ & 0,2102 & - & $-0,2183$ & - & 0,1977 & $-0,67$ \\
\hline$\Delta$ LnGinit-2 $_{\mathrm{t}}$ & - & - & 0,3974 & - & - & - & $-0,4818 *$ & $-2,09$ \\
\hline$\Delta$ LnGinit-3 $_{\mathrm{t}}$ & - & - & 0,1541 & - & - & - & $-0,8587 * * *$ & $-3,34$ \\
\hline$\Delta$ LnGini $_{\mathrm{t}-4}$ & - & - & 0,1699 & - & - & - & $-0,9479 * k *$ & $-3,56$ \\
\hline$\Delta L_{n G i n i} i_{-5}$ & - & - & $0,4268^{* *}$ & - & - & - & $-1,0538^{* * *}$ & $-3,93$ \\
\hline$\Delta$ LnGini $_{\mathrm{t}-6}$ & - & - & 0,33825 & - & - & - & $-0,6024^{*}$ & $-1,96$ \\
\hline$\Delta$ LnGinit-7 & - & - & - & - & - & - & $-0,0018$ & $-0,01$ \\
\hline$\Delta$ LnGinit-8 & - & - & - & - & - & - & $-0,1164$ & $-0,55$ \\
\hline d1984 & 0,0161 & $-1,6$ & - & - & $0,0424^{* *}$ & $-2,9$ & - & - \\
\hline $\mathrm{MCE}$ & $\begin{array}{c}- \\
0,3187 * *\end{array}$ & $-2,2$ & $-0,5473^{*}$ & $-2,4$ & 0,70477** & $-3,3$ & $-0,2664^{*}$ & $-2,07$ \\
\hline $\bar{R}^{2}$ & 0,2741 & - & 0,1808 & - & 0,3957 & - & 0,6149 & - \\
\hline Estatística F & 2,8313 & - & 1,5819 & - & 4,9112 & - & 3,875 & - \\
\hline D. Watson & 1,5978 & - & 1,8232 & - & 1,5978 & - & 2,5469 & - \\
\hline J arque-Bera & 1,7646 & - & 0,5363 & - & 1,7646 & - & 7,4059 & - \\
\hline White & 23,6567 & - & 0,1466 & - & 23,6567 & - & 4,3337 & - \\
\hline
\end{tabular}

Fonte: Elaboração própria a partir do software estatístico E-Views 7.0.

Akaike, obedecendo assim, a regra de seleção do número ótimo de defasagens partindo de um modelo geral para chegar a um modelo específico.

11 Também, foi estimado um modelo ADL $(6,7)$ tendo Gini como variável dependente e PIB como variável independente, Entretanto, ao estimar esse modelo, apesar de um $\mathrm{R}^{2}$ ajustado de 0,2467 e dos sinais da maioria dos parâmetros estarem conforme o esperado, encontrou-se que os parâmetros não são estatisticamente significantes. 
Os sinais dos parâmetros das variáveis PIBpc em primeira diferença com as defasagens são negativos, sugerindo que o crescimento econômico no período anterior reduz o crescimento contemporâneo, porém, somente o parâmetro da variável com uma defasagem é estatisticamente significativo. Os sinais dos parâmetros da primeira diferença de Gini, em sua maioria, negativos, estão conforme o esperado: variações positivas na concentração de renda no passado afetam negativamente o crescimento contemporâneo. Somente os parâmetros da primeira, sétima e oitava defasagens da primeira diferença de Gini não são estatisticamente significativos. Mediante a análise dos resíduos dos modelos $\operatorname{ADL}(6,3)$ e $\mathrm{ADL}(5,8)$ foi possível constatar que seguem uma distribuição normal (J arque-Bera), são não autocorrelacionados (teste Lagrange Multiplier de Breusch-Godfrey) e, também, são homocedásticos conforme o teste de White.

No que tange à causalidade no sentido de Granger, primeiramente, considerando a modelagem VAR, ao testar a hipótese de que o coeficiente da variável PIB na sua primeira diferença e com uma defasagem é igual a zero na equação da variável Gini como a dependente, constatou-se que não se pode rejeitar a hipótese nula de que $\Delta$ PIB não causa, no sentido de Granger, $\Delta$ Gini. Da mesma forma, na equação do PIB como a variável dependente, a hipótese de que o coeficiente da variável Gini na sua primeira diferença e com uma defasagem seja igual a zero não pode ser rejeitada. No entanto, ao considerar ambas as variáveis na sua primeira diferença e com seis defasagens, verifica-se bicausalidade da desigualdade para o PIB e do PIB para a desigualdade de renda, conforme TABELA 2.

TABELA 2 - RESULTADOS DOS TESTES DE WALD, CAUSALIDADE DE GRANGER, MODELOS VAR, VEC E ADL

\begin{tabular}{lccc}
\hline \multicolumn{1}{c}{ Hipótese nula } & Modelo & Probabilidade & Decisão \\
\hline$\Delta$ LnPIBpct $-1 \rightarrow \Delta$ LnGini & VAR & 0,3159 & Não causalidade \\
$\Delta L n P I B p c_{\mathrm{t}-6} \rightarrow \Delta$ LnGini & VEC & 0,0786 & Causalidade \\
$\Delta L n$ Gini $\rightarrow \Delta$ LnPIBpct-1 & VAR & 0,4044 & Não causalidade \\
$\Delta L n$ Gini $\rightarrow \Delta$ LnPIBpct-6 & VEC & 0,0003 & Causalidade \\
$\Delta L n$ Gini $\rightarrow \Delta$ LnPIBpc & ADL(5,8) & 0,0092 & Causalidade \\
$\Delta L n P I B p c \rightarrow \Delta$ LnGini & ADL $(6,3)$ & 0,0542 & Causalidade \\
\hline
\end{tabular}

Fonte: Elaboração própria.

No caso dos modelos ADL, para testar a causalidade de Granger, utilizou-se o teste de Wald para o modelo ADL $(5,8)$ tendo como variável dependente, a primeira diferença do PIB. Segundo esse teste, não foi possível rejeitar a hipótese nula de que as variações em Gini precedem temporalmente as variações no PIBpc, ao nível de significância estatística de 1\%. Portanto, existe uma causalidade unidirecional do Gini para o PIB, pois, os coeficientes estimados do Gini defasados em oito lags mais o mecanismo de correção de erros (MCE) são, conjuntamente, estatisticamente diferentes de zero. Logo, no Brasil, para o período 1976-2012, é possível afirmar que as variações na 
desigualdade precedem temporalmente as variações no crescimento econômico. Assim como no modelo ADL $(5,8)$, também se testou a causalidade de Granger do modelo ADL $(6,3)$. Conforme o teste de Wald, variações no PIBpc Grangercausa variações na desigualdade de renda ao nível de significância de $5 \%$.

Diante desse resultado obtido através do modelo ADL é possível afirmar que existe bicausalidade do Gini para o PIB e do PIB para o Gini. No Brasil, para o período 1976-2012, as variações na desigualdade de renda precedem temporalmente as variações no crescimento econômico e, também, que as variações no crescimento econômico precedem temporalmente as variações na desigualdade. Esse resultado difere daquele encontrado por Risso, Punzo e Carrera (2013) em suas estimativas para o México, pois, o teste de Granger apontou causalidade unidirecional do PIB per capita para o Gini.

A relação de causalidade da desigualdade de renda para o crescimento encontrada nas estimativas do modelo ADL $(5,8)$ sugere a existência de efeitos negativos da desigualdade para o PIB per capita. Essa constatação está em consonância com os canais de transmissão desses efeitos sobre o crescimento econômico e os respectivos modelos teóricos discutidos na revisão de literatura, tais como, por exemplo, os de imperfeições no mercado de crédito, o de economia política, o de conflito social e os de diferencial de fertilidade entre ricos e pobres.

Já a segunda relação de causalidade sugerida pelo modelo ADL $(6,3)$, do crescimento para a desigualdade, sugere a existência efeito positivo das variações do PIB per capita para a desigualdade, principalmente, ao considerar defasagem de quatro lags da variável explicativa. Esses resultados estão de acordo com os de Alesina e Rodrik (1991), Persson e Tabellini (1994), Li e Zou (1998), Forbes (2000) ou o efeito do crescimento sobre a desigualdade presente em, por exemplo, Dollar e Kraay (2002), Lopez (2006), Chambers (2007). Mas, poucos desses trabalhos abordaram esses efeitos simultaneamente. Um deles foi o de Lin, Huang e Yeh (2009) denominado "J oint determinations of inequality and growth".

O canal das imperfeições no mercado de crédito é um dos mecanismos de transmissão dos efeitos negativos da desigualdade de renda sobre o crescimento econômico que teve maior relevância durante o período da ditadura militar (1964-1985) por causa do favorecimento de grupos específicos que tiveram acesso facilitado aos empréstimos, financiamento habitacional e, também, ao mercado de ações. Mais recentemente, tem havido políticas públicas que também melhoraram esse acesso, como por exemplo, o Programa de Fortalecimento da Agricultura Familiar (PRONAF) ${ }^{12}$ e o programa de microcrédito ${ }^{13}$ que subsidia as taxas de juros para a tomada de empréstimos.

12 O PRONAF foi criado pelo Decreto no 1.946 de 28 de junho de 1996 com o objetivo de promover o desenvolvimento sustentável do segmento rural, aumentando a capacidade produtiva, a geração de empregos e melhoria de renda.

13 O Programa Nacional de Microcrédito Produtivo Orientado (PNMPO) foi instituído pela Lei no 11.110 de 2005 no âmbito do Ministério do Trabalho e do Emprego. Segundo dados desse Ministério, em 2013, o programa ofertou cerca de R\$ 9 bilhões atendendo a mais de 5 milhões de clientes. 
Logo, é bastante provável que o canal do mercado de crédito imperfeito mesmo com a reformulação do sistema financeiro ocorrida no conjunto de reformas institucionais do Plano de Ação Econômica do Governo (PAEG) durante a ditadura militar e com as políticas públicas mais recentes, continua sendo um importante mecanismo para explicar o efeito da desigualdade sobre o crescimento. O canal da redistribuição sugere que a desigualdade de renda é combatida com políticas redistributivas que são financiadas com impostos que por sua vez, causam distorções na economia, desestimulando o crescimento econômico. Segundo o Ministério da Fazenda, a carga tributária brasileira atingiu o patamar de 35,95\% no ano 2013, um salto de três pontos percentuais em relação ao ano 2004 quando teve início no Brasil, a transferência de renda de uma forma mais ampla, sistematizada e condicionada mediante o programa Bolsa Família ${ }^{14}$.

Segundo Skidmore (1990) o conflito social no Brasil não é um assunto que tenha causado grandes efeitos no crescimento econômico enquanto um canal e mesmo durante o período da ditadura militar não houve evidências de circunstâncias similares como uma guerra civil e explosão de motins políticos em excesso. Num sentido diferente de conflito social, Sachsida et al. (2010) estudaram a relação entre criminalidade e desigualdade de renda no Brasil e constataram que o elevado grau de desigualdade estimula o comportamento criminal, no entanto, refutaram a visão tradicional de que a pobreza afeta o crime violento. Baseandose nos testes de causalidade de Granger, não encontraram evidências de que a criminalidade causa a desigualdade. Considerando os argumentos do modelo teórico do conflito social enquanto mecanismo de transmissão da desigualdade de renda para o crescimento econômico, pode-se dizer que, recentemente, dada a amenização da perda do poder de compra das famílias mais pobres, a estabilização dos preços, a política de valorização do salário mínimo e, também, os benefícios dos programas de transferência de renda, houve aumento do custo de oportunidade para o desenvolvimento de atividades criminais, desestimulando-as e, consequentemente, melhorando o desempenho econômico. Conforme Potter et al. (2010) e Muniz (2009), o Brasil tem experimentado um forte declínio na taxa de fecundidade ao longo do período 1960 a 2000, mas esse não foi bem distribuído entre diferentes grupos de renda.

\section{Considerações finais}

Para responder à questão norteadora desta pesquisa, utilizou-se de duas abordagens econométricas distintas: a modelagem VEC e, também, os modelos autorregressivos de defasagens distribuídas (ADL). Para o caso brasileiro, considerando um período de quase quatro décadas, independentemente da

${ }^{14}$ A Lei no 10.836 de 9 de janeiro de 2004 unificou e ampliou diversos programas até então existentes, como por exemplo, o Programa Nacional de Renda Mínima vinculada à Educação (Bolsa Escola/ 2001), o Cadastramento Único do Governo Federal (2001), o Programa Nacional de Renda Mínima vinculada à Saúde (Bolsa Alimentação/ 2001), o Programa Auxílio-Gás (2002) e o Programa Nacional de Acesso à Alimentação (Fome Zero/ 2003). 
técnica utilizada, constatou-se a existência de uma relação de longo prazo entre crescimento econômico e desigualdade de renda, caracterizada por ser bicausal.

No Brasil, a desigualdade de renda prejudica o crescimento econômico por causa da inércia da desigualdade e, também, o crescimento econômico revelouse como instrumento importante para a redução da desigualdade de renda. Portanto, confirmou-se a hipótese inicial deste trabalho de que existe uma relação de longo prazo entre distribuição e crescimento e que, enquanto o crescimento econômico afeta positivamente a distribuição, essa, por sua vez, afeta negativamente o crescimento.

A relação causal, no sentido de Granger, encontrada no modelo autorregressivo de defasagens distribuídas respalda a orientação da política econômica brasileira adotada recentemente, principalmente a partir da implantação do Plano Real com foco no objetivo de estabilizar os preços e distribuir a renda via programas de transferências de renda e, também, mediante políticas públicas de inclusão social.

Entretanto, desde a implantação do Plano Real, há tendência de redução da concentração de renda no Brasil por causa do aumento do poder aquisitivo dos brasileiros mediante o combate à inflação, políticas de valorização do salário mínimo e de transferências de renda, entre outros fatores importantes. No entanto, ao que tudo indica, a atividade econômica não tem reagido positivamente à diminuição da desigualdade, provavelmente, por causa da componente inercial. Diante desse fato, o estudo sobre a relação entre distribuição e crescimento não se esgota neste trabalho, daí então, a necessidade de se aprofundar no estudo da relação desse binômio. Esse aprofundamento pode ocorrer, por exemplo, com a investigação dos canais de transmissão dos efeitos indiretos da desigualdade sobre o crescimento econômico, privilegiando também, o aspecto regional diante da característica de heterogeneidade da economia brasileira. Sugere-se também o estudo das determinações conjuntas da desigualdade de renda e do crescimento econômico mediante modelos de equações simultâneas em que se possa fazer o devido controle dos efeitos através da inclusão de variáveis capazes de captar a estabilidade macroeconômica.

Em termos de proposição de política econômica, as simulações das funções resposta ao impulso sugerem que o crescimento econômico é um instrumento importante para reduzir a desigualdade de renda no longo prazo. Confirmou-se o efeito negativo da desigualdade de renda e, portanto, a concentração de renda não pode ser considerada como sendo uma estratégia adequada para promover o crescimento econômico no longo prazo. As estratégias de redução da desigualdade e aceleração do crescimento econômico devem ser planejadas levando-se em consideração o contexto do longo prazo.

Ao refletir sobre a realidade brasileira com base nos modelos teóricos que tratam dos canais de transmissão da desigualdade sobre o crescimento econômico, essas estratégias devem estar relacionadas com a melhora contínua do acesso ao mercado de crédito para as famílias mais pobres terem a oportunidade de colocar seus projetos de investimento em prática; focalização ainda mais intensa das famílias a serem beneficiadas pelos programas de transferência de renda e pelas políticas públicas de inclusão social; 
desenvolvimento de políticas públicas e fortalecimento das instituições para amenização do conflito social; redução das incertezas e criação de um ambiente permeado de garantia dos direitos de propriedade.

Analisando a tendência declinante da taxa de fertilidade brasileira ao longo dos últimos anos, destaca-se que os canais da redistribuição e do diferencial de fertilidade provavelmente não são importantes para explicar o efeito negativo da desigualdade de renda sobre o crescimento econômico no Brasil. Isso implica que o baixo ritmo de crescimento da economia brasileira não está relacionado com as políticas redistributivas e que essas políticas, por sua vez, não podem ser condenadas por estimular o aumento do número de filhos nas famílias mais pobres, pois, o diferencial de fertilidade entre o rico e o pobre no Brasil está diminuindo cada vez mais.

\section{Referências}

Aghion, P. \& Howitt, P. (1998). Endogenous growth theory. (1 ed.). Cambridge: MIT Press.

Alesina, A. \& Rodrik, D. (1991). Distribution polities and economic growth. Quarterly J ournal of Economics. 109 (2), pp. 465-490.

Barro, R. J . (2000). Inequality and growth in a panel of countries. Quarterly J ournal of Economic Growth. 5(1), pp. 5-32.

Benabou, R. (1996). Inequality and growth. In: Bernanke, B.S \& Rotemberg, J eds. NBER Macroeconomics Annual. Cambridge: MIT Press.

Black, D. (1948). The decisions of a committee using a special majority. Econometrica: J ournal of the Econometric Society. 1(1), pp. 245-261.

Bourguignon, F. (1981). Pareto superiority of unegalitarian equilibria in stiglitz'model of wealth distribution with convex saving function. Econometrica: J ournal of the Econometric Society. 1(1), pp. 1469-1475.

Brasil. Decreto no 1946 de 28 de junho de 1996. Cria o Programa Nacional de Fortalecimento da Agricultura - PRONAF e dá outras providências. Disponível em: http://www.planalto.gov.br/ccivil 03/decreto/D1946.htm. [Acesso em 05 Julho 2016].

Brasil. Lei ordinária no 10.836 de 9 de janeiro de 2004. Cria o Programa Bolsa Família e dá outras providências. Disponível em: http://www.planalto.gov.br/ccivil 03/ ato2004-2006/2004/lei/l10.836.htm. [Acesso em 05Julho 2016].

Brasil. Lei ordinária no 11.110 de 25 de abril de 2005. Institui o Programa Nacional de Microcrédito Produtivo Orientado - PNMPO. Disponível em: http:// www.planalto.gov.br/ccivil 03/ ato2004-2006/2004/lei/l10.836.htm. [Acesso em 05J ulho 2016].

Chambers, D. (2007). Trading places: does past growth impact inequality?. J ournal of development economic. 82(1), pp. 257-266. 
Dickey, D. A. \& Fuller, W. A. (1979). Distribution of the Estimators for Autoregressive Time Series with a Unit Root. Journal of the American Statistical Association, 74 (366), pp. 427-31.

Ehrhart, C. (2009). The Effects of Inequality on Growth: A Survey of the Theoretical and Empirical Literature. ECINEQ Working Papers, 107.

Elliot, G., Rothemberg, T. \& Stock, J. H. (1996). Efficient Tests for an Autoregressive Unit Root. Econometrica, 64 (4), pp. 813-836.

Enders, W. (2004). Applied econometric time series. (2 ed.). Wiley Series in Probability and Statistics. Nova York: International Edition - Wiley.

Engle, R. F. \& Granger, C.W.J. (1987). Co-integration and error-correction: representation, estimation, and testing. Econometrica, 55, pp. 251-76.

Fishlow, A. (1975). A distribuição de renda no Brasil. In: Tolipan, T. (orgs.). A controvérsia sobre a distribuição de renda e desenvolvimento. Rio de J aneiro: Zahar, pp. 159-189.

Forbes, K. (2000). A reassessment of relationship between inequality and growth. American Economic Review, 90 (4), pp. 869- 886.

Furtado, C. (1982). Análise do "modelo" brasileiro. (7. Ed). Rio de Janeiro: Civilização Brasileira S.A.

Gadelha, S. R. de B. (2011). Causalidade temporal entre receita e despesa governamentais. Revista Análise Econômica. Porto Alegre, 29 (56), pp. 109130.

Galor, O. \& Zeira, J . (1993). Income distribution and macroeconomics. Review of Economic Studies, 60, pp.35-52.

Galor, O. \& Moav, O. (2004). From physical to human capital accumulation: inequality and the process of development. Review of Economic Studies, 1(71), pp. 1001-1026.

Granger, C. W. J . (1989). Investigating Causal Relations by Econometric Models and Cross-Spectral Methods, Econometrica, 37, pp. 424- 438.

Hoffmann, R. \& Duarte, J . C. (1972). A distribuição da renda no Brasil. Revista de Administração de Empresas, 12 (2), pp. 46-66.

Iradian, G. (2005). Inequality, poverty and growth: cross-country evidence. International Monetary Fund Working Paper, 5(28).

Johansen, S. (1988). Statistical analysis of cointegration vectors, Journal of Economic Dynamics and Control, 12, pp.231-254. 
Johansen, S., Mosconi, R. \& Nielsen, B. (2000). Cointegration analysis in the presence of structural breaks in the deterministic trend, Econometrics J ournal, 3, pp. 216-249.

Jones, C. I. (1995). Time series tests of endogenous growth models. The Quarterly J ournal of Economics, pp. 495-525.

Kaldor, N. (1957). A model of economic growth. The economic journal, 67(268), pp. 591-624.

Keynes, J. M. (1920). The Economic Consequences of the Peace. Macmillan.

Kuznets, S. (1955). Economic Growth and Income Inequality. American Economic Review, 45, pp. 1-28.

Langoni, C. G. (1973). Distribuição de renda e desenvolvimento econômico do Brasil. Rio deJ aneiro: Expressão e Cultura.

Lanne, M., Lutkepohl, H. \& Saikkonen, P. (2002). Comparison of unit root tests for time series with level shifts. Journal of time series analysis, Wiley Online Library, 23(6), pp. 667-685.

Li, H.; Zou, H. (1998). Income inequality is not harmful for grouth: theory and evidence. Review of Development Economics, 2(3), pp.318-334.

Muniz, J. O. (2009). Differential reproduction, poverty and the dynamics of inequality in Brazil, 1980-2000. Madison: tese de doutorado em Sociologia, Universidade de Wisconsin.

Ng, S.; Perron, P. (2001). Lag length selection and the construction of unit root tests with good size and power. Econometrica, 69 (6), pp. 1519-1554.

Okun, A.M. (1975). Equality and efficiency: The big trade-off. Brookings Institution, Washington, DC.

Perotti, R. (1996). Democracy, income distribution and growth: What the data say. J ournal of Economic Growth, 1(1), pp. 149-187.

Perron, P. (1989). The Great Crash, the Oil Shock, and the Unit Root Hypothesis. Econometrica, 57, pp. 1361-1401.

Persson, T. \& Tabellini, G. (1994). Is inequality harmful for growth? Theory and evidence. American Economic Review, 84, pp. 600-621.

Phillips, P. \& Perron, P. (1988). Testing for a Unit Root in Time Series Regression. Biometrika, 75(2), pp. 335-346.

Piketty, T. (2014). O capital no século XXI. Rio de Janeiro: Intrínseca. 
Potter, J . E., Schmertmann, C. P., Assunção, R. M. \& Cavenaghi, S. M. (2010). Mapping the timing, pace, and scale of the fertility transition in Brazil. Population and development review, 36(2), pp.283-307.

Risso, W.A., Punzo, L. F. \& Carrera, E. J. S. (2013). Economic growth and income distribution in Mexico: A cointegration exercise. Economic Modelling, 35, pp. 708- 714 .

Sachsida, A., Gutierrez, M. B. S, De Mendonça, M. J. C. \& Loureiro, P. R. A. (2010). Inequality and criminality revisited: further evidence from Brazil. Empirical Economics, Springer, 39(1), pp. 93- 109.

Said, S., Dickey, D. A. (1984). Testing for unit roots in autoregressive-moving average models of unknown order. Biometrika, 71.

Saikkonen, P. \& H. Lütkepohl. (2002). Testing for a unit root in a time series with a level shift at unknown time. Econometric Theory, 18, pp. 313-348.

Senna, J J J. (1976). Escolaridade, experiência no trabalho e salário no Brasil. Revista Brasileira deEconomia, 30(2), pp. 163-193.

Shin, I. Income inequality and economic growth. (2012). Economic Modelling, 29, pp. 2049- 2057.

Singer, P. (1975). Desenvolvimento e repartição da renda no Brasil. In: Tolipan; T. (orgs.). A controvérsia sobre a distribuição de renda e desenvolvimento. Rio de J aneiro: Zahar, pp. 159-189.

Skidmore, T. E. (1990). The politics of military rule in Brazil, 1964-1985. Oxford University Press.

Smith, A. (1776). The wealth of nations, 1937.

Stock, J . H.; Watson, M. W. (2004). Econometria. São Paulo: Addison Wesley.

Tavares, M. \& Assis, J. C. (1985). O grande salto para o caos: a economia política e a política econômica do regime autoritário. Rio de Janeiro: Jorge Zahar.

Zhou, X. \& Li, K. W. (2011). Inequality and development: Evidence from semiparametric estimation with panel data. Economics Letters, 113:203- 207. 